Natural Hazards and Earth System Sciences (2003) 3: 637-645

(C) European Geosciences Union 2003

Natural Hazards and Earth System Sciences

\title{
Neutron flux variations near the Earth's crust. A possible tectonic activity detection
}

\author{
B. M. Kuzhevskij, O. Yu. Nechaev, E. A. Sigaeva, and V. A. Zakharov \\ Skobeltsyn Institute of Nuclear Physics of Lomonosov Moscow State University, Vorobjovy 3 Gory, Moscow, 119899, Russia
}

Received: 19 February 2002 - Revised: 11 July 2002 - Accepted: 10 January 2003

\begin{abstract}
The present work contains some results of observations of neutron flux variations near the Earth's surface. The Earth's crust is determined to be a significant source of thermal and slow neutrons, originated from the interaction between the nuclei of the elements of the Earth's crust and the atmosphere and $\alpha$-particles, produced by decay of radioactive gases (Radon, Thoron and Actinon). In turn, variations of radioactive gases exhalation is connected with geodynamical processes in the Earth's crust, including tectonic activity. This determined relation between the processes in the Earth's crust and neutrons' flux allow to use variations of thermal and slow neutrons' flux in order to observe increasing tectonic activity and to develop methods for short-term prediction of natural hazards.
\end{abstract}

\section{Introduction}

Composition, variations in time and spatial distribution of the particles of nuclear radiation in the lower atmosphere have been studied for about 50 years.

Many scientists studied the energy spectrum and the altitudinal, latitudinal and longitudinal distribution of neutrons' fluxes in the lower atmosphere (Gorshkov et al., 1966;). The results of these studies lay in the following frames: neutron component of nuclear irradiation in the lower atmosphere is produced during the interaction between high-energy primary particles of cosmic origin and nuclei of the elements existing in the lower atmosphere. Vertical control traverse of the counting rate of neutrons has a maximum at the altitude of $16-17 \mathrm{~km}$, while near the Earth's surface the most part of neutron flux consists of the particles of thermal energies. Speaking about vertical control traverse of neutrons counting rate, it's necessary to take into consideration the average elevation rate of the balloon, which is about $300 \mathrm{~m} / \mathrm{min}$. So, during the studies of altitudinal distribution of neutrons in the

Correspondence to: B. M. Kuzhevskij

(bmk@srd.sinp.msu.ru) lower atmosphere balloon flies through the near-Earth layer up to the altitudes of several kilometers in 7-8 min. Counting rate in the near-Earth surface layer of the atmosphere is low and in the case of low detector's lumen the most part of the scientists, as a rule, averaged neutrons' counting rate during 5 min. Such averaging does not allow to conclude anything about detailed character of the distribution (and consequently about the sources) of neutron component in the lower atmosphere up to the altitude of several kilometers.

In 1990 we launched a balloon with neutrons' detector near Apatity, Kol'sky peninsula. The total sensitive area of the detector was $1500 \mathrm{~cm}^{2}$. Such lumen output of the device allowed to get reliable information per minute. Figure 1 presents the vertical control traverse of neutrons' counting rate, which, in general, is in agreement with the results of other scientists. Detailed vertical control traverse of neutrons' counting rate up to the altitude of $5 \mathrm{~km}$ is presented at small axes in Fig. 1. Altitude dependence if described with a function:

$$
N(h)=\left\{\begin{array}{cl}
50.3 h^{2.1} \exp (-0.03 h) & 2 \leq h<8 \\
50.3 h^{2.1} \exp (-0.03 h) & \\
+\left(1+\frac{h_{\max }-h}{5}\right) h^{3} & 8 \leq h \leq h_{\max } \\
250(116-3 h) & h>18
\end{array}\right.
$$

where $h$ is in kilometer scale, $h_{\max }=17 \mathrm{~km}$.

It's easy to see that approximately up to the altitude of $2 \mathrm{~km}$, the counting rate essentially did not depend on the altitude.

Later the device, which allowed to detect neutrons' fluxes simultaneously in two vertical directions, from and to the Earth, was launched with balloons in Dolgoprudny, Moscow region (18 June 1991) and in Ryl'sk, Kursk region (13 February 1992). During the experiment in Dolgoprudny, which took place in summer (1991), the vertical control traverse of neutrons' counting rate was not found up to approximately $2 \mathrm{~km}$. In Ryl'sk experiment, which took place in winter (1992), freedom of neutrons' counting rate from the altitude was not distinctly detected (Fig. 2). During the first 


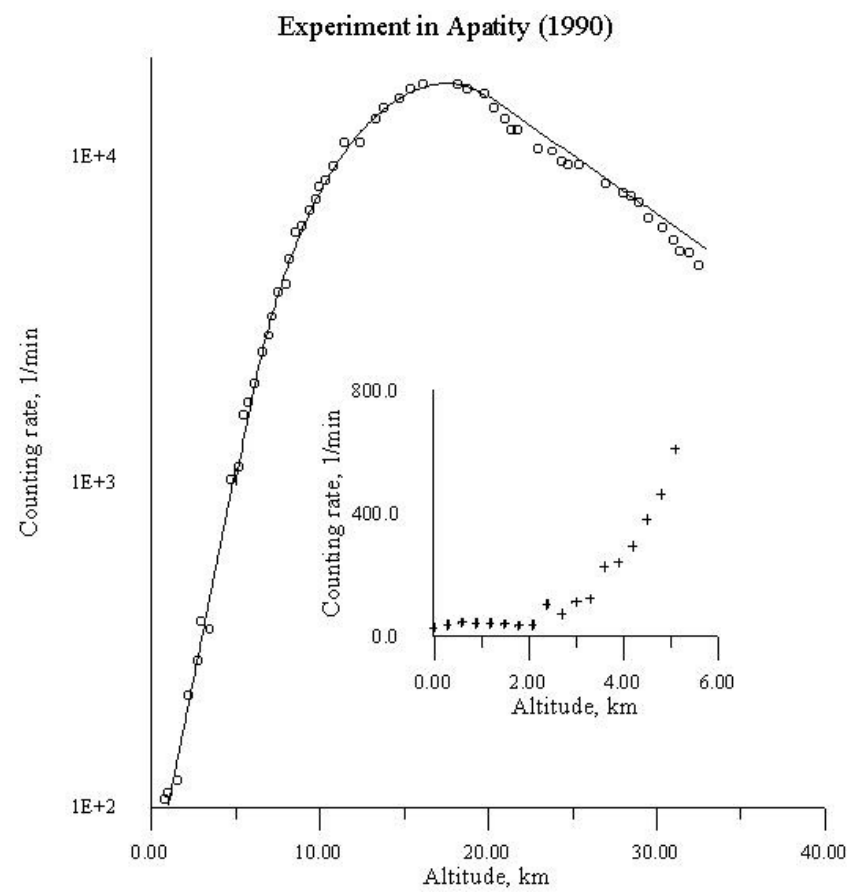

Fig. 1. Altitude dependence of neutrons' counting rate, obtained in the experiment, arranged in 1990 during the flight of an aerostat over Apatity, Kol'sky peninsula. Small circles indicate experimental data.

flight up to the altitude of several kilometers, the equipment determined an anisotropy of neutrons' flux, which showed that value of neutron flux from the Earth was over the value of neutron flux to the Earth (Kuzhevskij et al., 1995; Kuzhevskij, 2000).

In the laboratory of penetrating radiation of INP MSU, a special equipment was developed and manufactured for detailed studies on the origin of neutron radiation near the Earth's surface. This equipment allows to obtain continuous information regarding the registration of fluxes of neutrons and charged particles. This equipment started operating from September 1992 and a number of the results of this experiment was presented in a previous study (Kuzhevskij et al., 1996).

\section{Experimental methods}

There are different physical principles and methods of processing the information obtained by the Detector of Nuclear Radiation of the Earth and it's Atmosphere DAIZA (Russian abbreviation) as follows:

Standard neutron counters SI-19N were used as detectors of neutron component. These counters had a shape of cylinder with length of $22 \mathrm{~cm}$ and diameter of $3.2 \mathrm{~cm}$. The sensitive area of the counter was $51 \mathrm{~cm}^{2}$. The counter was filled with ${ }^{3} \mathrm{He}$ gas pressurized at 4 atmospheres and it was operated in regime of corona discharge. Effective cross sec-

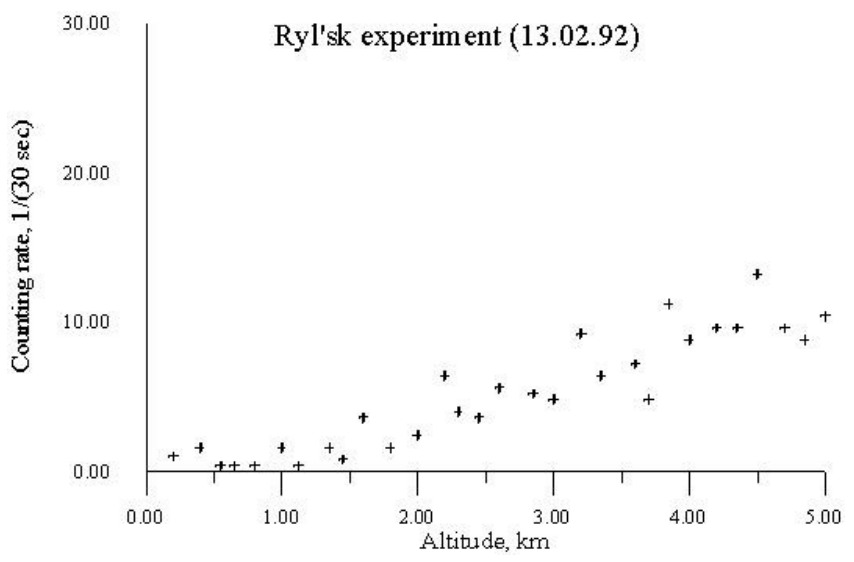

Fig. 2. Altitude dependence of neutrons' counting rate obtained in the experiment, arranged in 1992 during the flight of aerostat over Ryl'sk, Kursky region.

tion of the neutron captured reaction ${ }^{3} \mathrm{He}(\mathrm{n}, \mathrm{p}) \mathrm{T}$ with liberation of energy of $760 \mathrm{keV}$ is over $5000 \mathrm{bn}$, while other possible reactions (for instance, ${ }^{3} \mathrm{He}(\mathrm{n}, \gamma){ }^{4} \mathrm{He}$ and ${ }^{3} \mathrm{He}(\mathrm{n}, \mathrm{d}) \mathrm{d}$ ) have effective cross sections from dozens $\mu \mathrm{bn}$ up to hundreds of mbn. Stability tests of operation of neutron modules (12 such counters), which were held under special conditions (Kapitonov and Gabelko, 1990) in the laboratory, have shown that its stability was not lower than $1.5 \%$ and it was slightly changed at neutron's energy changes from heating up to $2 \mathrm{MeV}$. For such counters, the effectiveness of detection of neutrons in the ranges of thermal region up to energy of $100 \mathrm{eV}$ was 0.8 .

Currently, the neutron block consisted of two planes, including 40 counters each. Two identical detectors used in the device allowed to carry out research in different geometry and to study the energy distribution of neutrons by means of an absorbent (for instance, cadmium) and a retardant (for instance, plexiglass).

Counters SBT-10 (threshold for electrons was $E_{e} \geq$ $45 \mathrm{keV}$, for protons $E_{p} \geq 0.5 \mathrm{MeV}$ and for $\alpha$-particles $E_{\alpha} \sim 5 \mathrm{MeV}$ ) and two Geiger-Müller counters STS-6 were used for the detection of charged particles. One of the Geiger-Müller counters had an aluminum window $0.5 \mathrm{~cm}$ thick.

A scintillation crystal of $\mathrm{NaI}(\mathrm{Tl}) 7 \mathrm{~cm}$ long and $3 \mathrm{~cm}$ in diameter was used for the detection of $\gamma$-radiation. There were two energy thresholds: $E_{\gamma} \geq 50 \mathrm{keV}$ and $E_{\gamma} \geq$ $500 \mathrm{keV}$, which later were changed to $E_{\gamma} \geq 300 \mathrm{keV}$ and $E_{\gamma} \geq 2 \mathrm{MeV}$ in order to diminish background effects and to detect $\gamma-$ particles originated from the decay of radioactive gases.

Further, the authors mainly analyzed the information obtained by the neutron block. Information obtained by the measurement of charged particles component and $\gamma$-radiation will be discussed.

Similar to a stational unit DAIZA, there was also a mobile unit DAIZA-2, which contained 10 same neutron counters. 


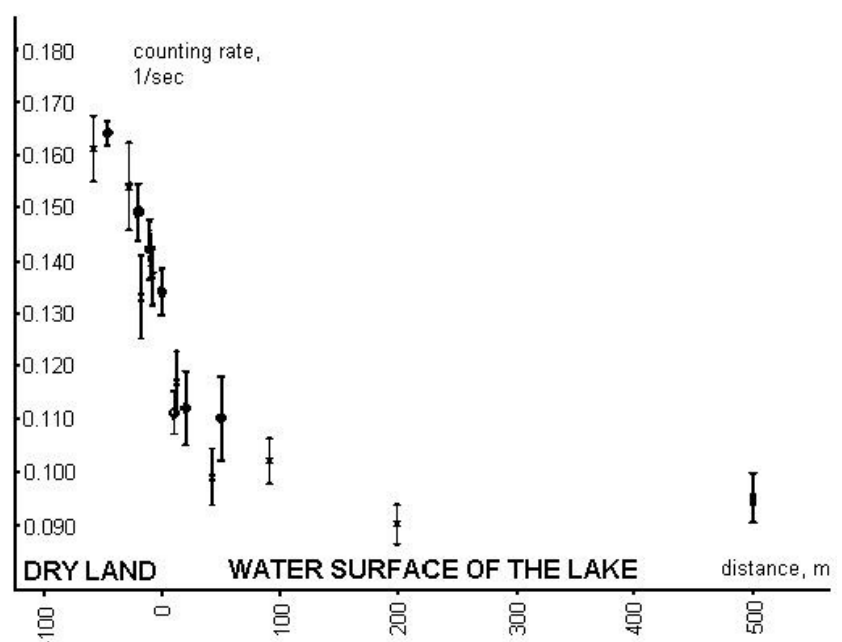

Fig. 3. Data on neutron flux in an experiment in the area of Seliger Lake.

Table 1. Counting rate of thermal and slow neutrons in soil in different locations in Russia obtained by means of DAIZA-2 project

\begin{tabular}{lc}
\hline Point of measurement & Counting rate per hour \\
\hline Vorobjovy Gory, Moscow & $414 \pm 7$ \\
Golitsyno, Moscow region & $475 \pm 5$ \\
Area of Seliger region, Tver' region & $579 \pm 4$ \\
\hline
\end{tabular}

It was provided with $3.2 \mathrm{MB}$ storage. By means of this plant the neutron flux in different locations in Moscow and Tver' regions was measured.

\section{Results}

Detailed studies on the energy distribution of neutrons on the basis of experimental data obtained with DAIZA unit in the Institute of Nuclear Physics of the Moscow State University, have shown, that near the Earth's crust over 70\% of neutrons provide energy $\leq 0.45 \mathrm{eV}$ (Beliaeva et al., 1999a, b).

In July - September 1999, there were carried out measurements on soil in different locations of the European part of Russia by means of DAIZA- 2 unit. The results of these measurements are summarized in Table 1.

This data substantially (for 10-15\%) differs, although galactic cosmic rays flux has varied not more than $1-2 \%$ for that period. According to experimental data, obtained by GOES- 8 satellite, there were observed small fluxes of protons with energy $E_{p}>10 \mathrm{MeV}$. By themselves, protons of such energy can't produce any variation of neutron flux at sea level. All given facts lead to the conclusion that neutron flux from the Earth's crust was determined by its chemical composition and structure.

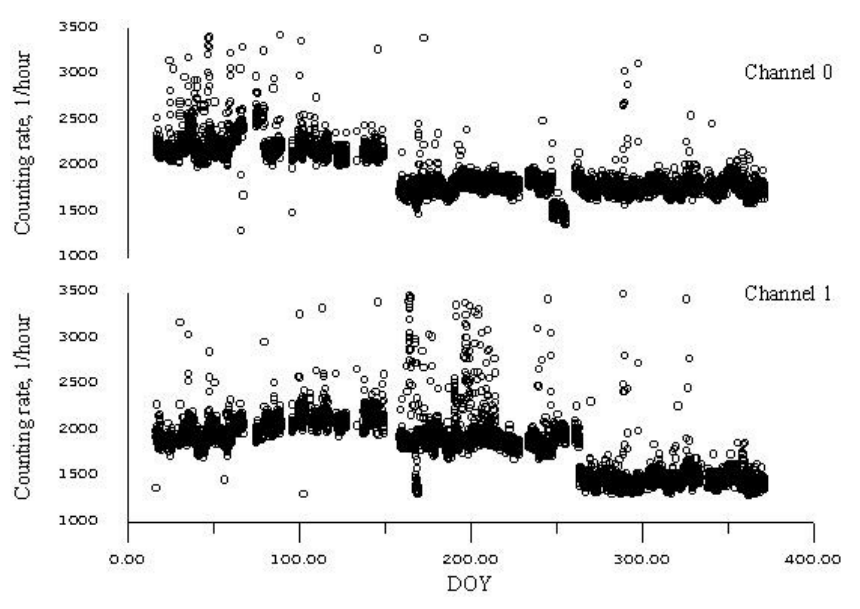

Fig. 4. The whole array of experimental data on neutron flux for the period started from 16 Jan. 1996 up to 31 Dec. 1996 (Channels 0 and 1 detected neutrons, consequently, from above and from below).

During the experiment taken place in the area of Seliger Lake (Fig. 3), it was found out that the total neutron flux above the dry land was 1.8 times larger than the total neutron flux above the water surface of the Lake at the distance of $200 \mathrm{~m}$ from the bank. Besides, during this experiment it succeeded to determine the neutron flux directed from the atmosphere to the Earth's surface. In Fig. 3, it's seen, that beginning from the distance of $200 \mathrm{~m}$, the total neutron flux did not change while moving from dry land to the center of the Lake. By this means, the total flux (its value is about $0.00018 \mathrm{~cm}^{-2} \mathrm{~s}^{-1}$ ) consisted only of neutrons coming from above, which were produced as a result of the nuclear interaction between cosmic rays and nuclei of the lower atmosphere elements.

In general all data, obtained in the foregoing experiments point to the fact that crust of the Earth is also a source of neutrons of low energies. This source was characterized by a wide range fluctuations of the flux both in time and in different regions.

\section{Anisotropy of neutron flux near the Earth's surface}

All obtained results lead to a need of more detailed studies of time variations of neutron flux from the Earth's surface. For detailed analysis, we used data obtained in 1996.

The whole data array was presented in Fig. 4. It's easy to see, that for different periods of measurements there was observed predominance of neutrons from different directions (to the Earth and from the Earth). At the same time, difference of neutrons' counting rates from these two directions was out of limits of statistical error. It's necessary to note, that even at this stage we can see that for the channel which detected neutrons from above, the most part of the counting rate splashes falls within the areas up to 100 and after 250 days of the year, which approximately conforms to 


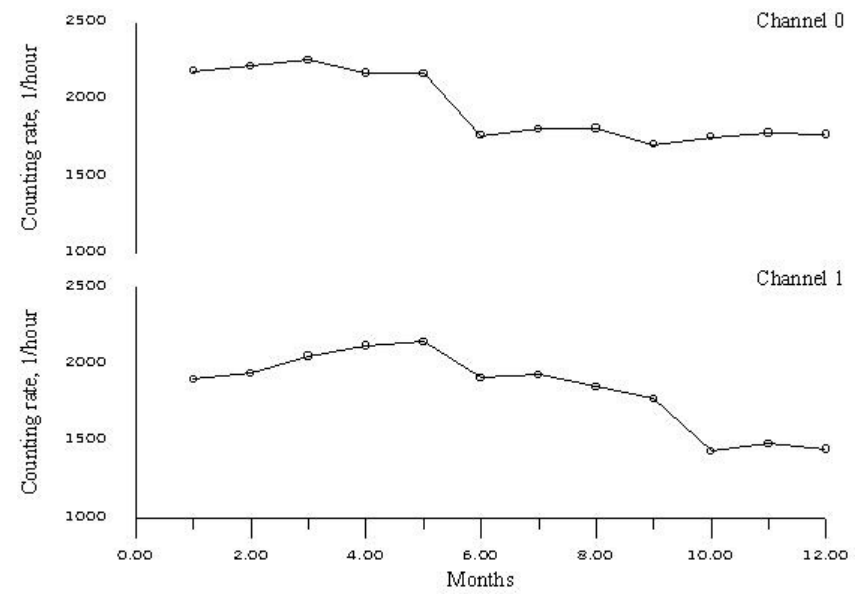

Fig. 5. Monthly mean experimental data of neutrons counting rate for quiet periods from 16 Jan. 1996 up to 31 Dec. 1996 (Channels 0 and 1 detected neutrons, consequently, from above and from below).

the period from October up to March, while for the channel which detects neutrons from below, the most part of the counting rate splashes falls within the area from 150 up to 250 days of the year, which approximately conforms to the period from June up to September.

To study changes of neutron flux anisotropy during the year, it is more suitable further to operate with monthly-mean neutrons counting rates. This data for two channels was presented in Fig. 5. During the averaging procedure we took into consideration only quiet periods of measurements, i.e. the periods when the counting rate was not more than $10 \%$ over the monthly-mean value. In 1996, about $80 \%$ of the observed cases were referred to the quiet periods of measurements, and for different months this part varied from 65 up to $97 \%$.

For this array, we counted out the ratio, $\mathrm{R}$ of neutron counting rates to the Earth and from the Earth and the anisotropy, $K_{a n}$, consequently. According to the formulas:

$$
\begin{aligned}
& R=\frac{N_{0}}{N_{1}} \\
& K_{a n}=\frac{N_{0}-N_{1}}{N_{0}+N_{1}} \times 100 \%
\end{aligned}
$$

where $N_{0}, N_{1}$ - are the counting rates of neutrons in the directions to the Earth and from the Earth, respectively.

The obtained results are presented in Fig. 6. We see that during the quiet periods of measurements, the value of anisotropy was up to $10 \%$, but at the same time the sign of anisotropy changed depending on month. Taking into account the values of errors, the anisotropy was clearly positive during the periods from January to March and from October to December. It means that during these periods of time, the neutron flux prevailed in the direction to the Earth. During the summer periods, or, taking into consideration the values of errors, in June and July, the situation was opposite: i.e. the anisotropy was negative and it means predominance

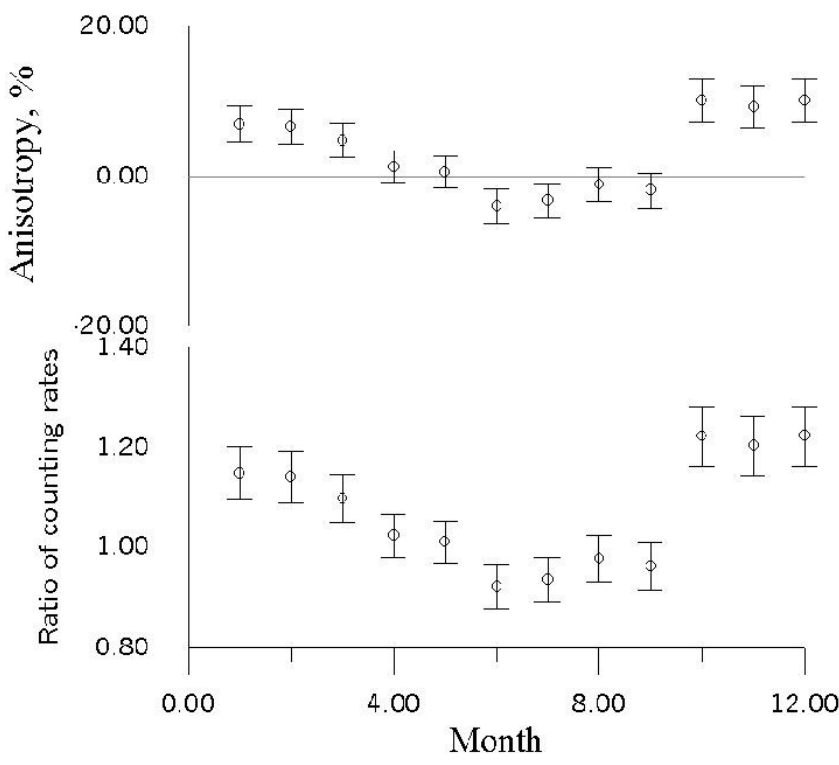

Fig. 6. Monthly-mean values of anisotropy and ratio of neutrons counting rates during the quiet periods from 16 Jan. 1996 up to 31 Dec. 1996

of neutron flux in the direction from the Earth. During the last periods (April-May and August-September) the value of anisotropy was close to zero, and we couldn't approve prevalence of neutron flux from one defined direction.

Analogous calculations were made for different years, when configuration of the plan was alike and there was a possibility for measurement of neutrons' fluxes to the Earth and from the Earth, separately.

Figure 7 presents the obtained monthly-mean anisotropy data for 1994-1997.

\section{Analysis of the results}

So, during the quiet periods of measurements, the value of neutron fluxes anisotropy was only several percents, although during the studied period (from January to December 1996) it changed its sign. In January-February, the flux to the Earth prevailed, while in June-July the situation changed and flux from the Earth prevailed. Next time the sign of the anisotropy changed in October, from which and up to the end of the year the flux to the Earth prevailed again. During the intermediate periods (March-May and August-September) there was no anisotropy of neutron fluxes, i.e. there was no predomination of one direction for the neutron fluxes.

Data analysis for different years (1994, 1995 and 1997) confirm such seasonal dependence of neutrons' anisotropy. Differences, observed for anisotropy for different years, can be explained by the unique character of each time period concerning many factors, including weather, solar and geophysical factors. 


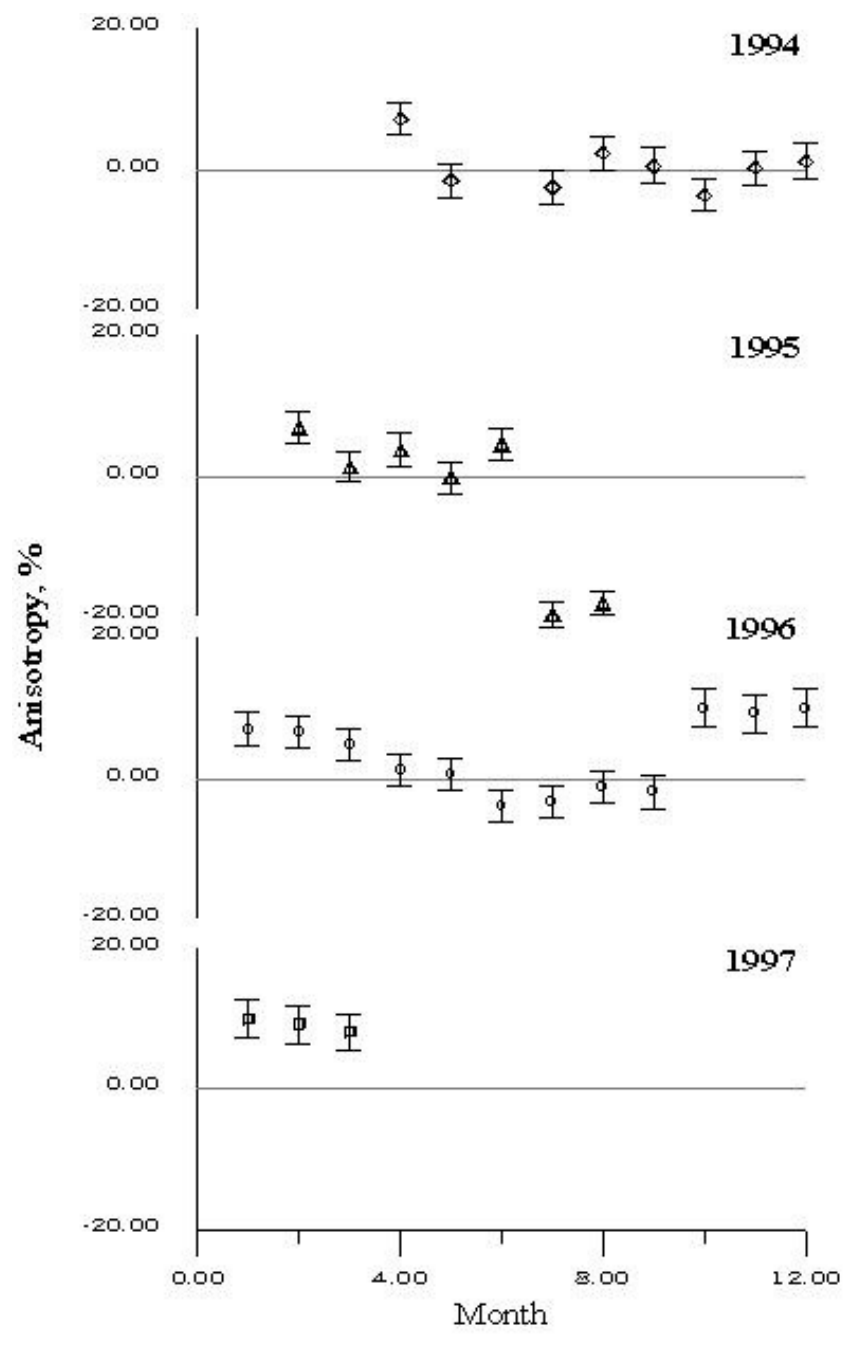

Fig. 7. Monthly-mean anisotropy data of neutrons counting rate for 1994-1997.

To explain such seasonal dependence of anisotropy it's necessary to take into consideration that the Earth as a source of thermal neutrons, plays double role. On the one hand, it reflects phenomena of the space. It means, that flux of highenergy particles coming to the Earth can produce thermal neutrons in its crust. On the other hand, splashes of neutron radiation are produced by $\alpha$-particles of radioactive isotopes of Radon by the interaction with nuclei of elements of the air and the Earth's crust. Of course, in the winter period, when the Earth's surface is steeped with water and covered with a layer of snow, the Earth's crust can't work as a source of thermal neutrons, because they will be absorbed before leaving the crust. Therefore, during this time the neutron flux in direction to the Earth must prevail, and we observed this fact. It concerns both the quiet periods of measurements and splashes of neutrons.

During the warm seasons, the Earth's crust is warmed up and begins to work both as a target for cosmic particles and as an independent source of neutrons.

\section{Radioactive gases, a source of the neutrons in the lower atmosphere}

According to Volodichev et al. (1992) we related the appearance of neutrons' splash during Sun's eclipse with the exhalation of Radon during deformation of the Earth's crust. But it is known that different radioactive gases (Radon, Thoron, Actinon) are exhaled continuously into the atmosphere from the Earth's crust. These gases are sources of $\alpha$-particles with energies from 5 up to $9 \mathrm{MeV}$. Nuclear interaction of these $\alpha$-particles with nuclei of the elements of the Earth's crust and the atmosphere result in production of neutrons. All these $(\alpha, n)$ reactions are threshold reactions. For nuclear reactions between $\alpha$-particles with mentioned above energies $(5-9 \mathrm{MeV})$ and the elements from the lower atmosphere (nitrogen, oxygen and carbon) effective cross-sections are about several mbn (Lingenfelter et. al., 1965; Kuzhevskij, 1985). For the Earth's crust besides these elements it's necessary to take into consideration aluminium and silicon isotopes. The reaction ${ }^{28} \mathrm{Si}(\alpha, n)$ has a threshold of about $9.2 \mathrm{MeV}$, but ${ }^{27} \mathrm{Al}(\alpha, n)$ has a threshold of $3 \mathrm{MeV}$. Reactions of other silicon isotopes, ${ }^{29} \mathrm{Si}(\alpha, n)$ and ${ }^{30} \mathrm{Si}(\alpha, n)$ have thresholds, consequently, of about 3 and $4 \mathrm{MeV}$.

Let's investigate the role of this source of neutrons quantitatively. If $N_{\alpha}(E)$ is the concentration of $\alpha$-particles with energy $E$ in the atmosphere, then the rate of neutrons' production in the unit of volume, $Q_{n}$ can be found as following:

$Q_{n}=\sum_{j, n} N_{j} N_{\alpha}(E) \sigma_{\alpha j}^{n}(E) v_{\alpha j}$,

where $N_{j}$ is the concentration of nuclei $j$ in the air, $\sigma_{\alpha j}^{n}(E)$ is the cross section of neutron's production by the interaction of $\alpha$-particle with nucleus $j, v_{\alpha j}$ is the relative velocity, which in fact coincides with $v_{\alpha}$, the velocity of $\alpha$-particle. Summation is made by all types of nuclei in the air (oxygen, nitrogen, etc.), by the energies of produced neutrons and by the energies of $\alpha$-particles.

The number, $N_{\alpha}$ of $\alpha$-particles, which are produced by the radioactive gases of Radon, can be found from the equation:

$\frac{d N_{\alpha}}{d t}+\lambda_{\alpha} N_{\alpha}=Q_{\alpha}$

where, $Q_{\alpha}$ is the rate of production of $\alpha$-particles in the unit of volume, $\lambda_{\alpha}=v_{\alpha} / R_{\alpha}$ defines the rate of $\alpha$-particles' descent, $v_{\alpha}$ is the velocity of $\alpha$-particle, $R_{\alpha}$ is the meanfree path of $\alpha$-particle in the air (for $E_{\alpha}=5 \div 9 \mathrm{MeV}, v_{\alpha} \approx$ $10^{9} \mathrm{~cm} / \mathrm{s}, R_{\alpha}=3 \div 9 \mathrm{~cm}$ ). Solution of the Eq. (4) looks like:

$N_{\alpha}(t)=\frac{Q_{\alpha}}{\lambda_{\alpha}}\left(1-e^{-\lambda_{\alpha} t}\right)$.

For the steady state condition, which is of the interest, we obtain:

$N_{\alpha}=\frac{Q_{\alpha}}{\lambda_{\alpha}}=\frac{Q_{\alpha} R_{\alpha}}{v_{\alpha}}$ 
Table 2. Rate of production $\left(Q_{\alpha}\right)$ of $\alpha$-particles in air

\begin{tabular}{lcc}
\hline Geographic location & $Q_{\alpha} \mathrm{cm}^{-3} \mathrm{~s}^{-1}$ & Remarks \\
\hline Czech Republic (Burian, 1966) & $\geq 2 \times 10^{-4}$ & Ground air \\
Belorus (Rudakov et al., 1995) & $2 \times 10^{-2}$ & $\begin{array}{c}\text { Data according three types of measurements. } \\
\alpha-\text { particles' flux was measured. }\end{array}$ \\
Pamir (Volodichev et.al., 2001) & $(1.5 \div 4.5) \times 10^{-3}$ & The measurements were made in summer. \\
London (Anderson et al., 1954) & $1.5 \times 10^{-3}$ & \\
$\begin{array}{l}\text { Region of uranium mines } \\
\text { (Serdukova, Kapitanov, 1975) }\end{array}$ & $5.6 \times 10^{-1}$ & \\
$\begin{array}{l}\text { Mean value over the land } \\
\text { (Serdukova, Kapitanov, 1975) }\end{array}$ & $1.5 \times 10^{-9} \div 3.3 \times 10^{-5}$ & \\
on Tn & $1.8 \times 10^{-6} \div 3.1 \times 10^{-5}$ & \\
on Rn & $1.8 \times 10^{-6}$ & \\
$\sum_{\text {min }}$ (Tn + Rn) & $6.4 \times 10^{-5}$ & \\
$\sum_{\text {max }}$ (Tn + Rn) & $3.7 \times 10^{-4} \div 7.4 \times 10^{-1}$ & Contents in the ground \\
Contents of Rn isotopes & & (Serdukova, Kapitanov, 1975) \\
\hline
\end{tabular}

Putting Eq. (3) into Eq. (1), we obtain the rate of neutrons' production:

$Q_{n}=\sum_{j, n} N_{j} Q_{\alpha} R_{\alpha} \sigma_{\alpha j}^{n}$

Then, the number of neutrons, $N_{n}$ is found by the equation:

$N_{n}=\frac{Q_{n}}{\lambda_{n}}=q_{n} \tau_{n}=\frac{\sum_{j, n} N_{j} Q_{\alpha} R_{\alpha} \sigma_{\alpha j}^{n}}{\lambda_{n}}$

where tn is the neutron's life span in the atmosphere (as calculated below $\tau_{n} \approx 0.2 \mathrm{~s}$ ), $\lambda_{n}=1 / \tau_{n}-$ is the rate of neutrons' descent in the atmosphere.

Using Eqs. (6) and (7), we study the contribution of radioactive gases in the production of number and flux of neutrons observed near the Earth's surface.

According to experimental data (Segre, 1995) the meanfree path $R_{\alpha}$ of $\alpha$-particles with energy $E_{\alpha}$ from 5 up to $9 \mathrm{MeV}$ changes from 3.5 to $8.9 \mathrm{~cm}$. The number of nuclei $N_{j}$ is defined, first of all, by nitrogen and oxygen, that is the Loschmidt number $N=2.687 \times 10^{19} \mathrm{~cm}^{-3}$.

The cross section, $\sigma_{\alpha j}^{n}$ for oxygen and nitrogen of $\alpha$-particles with energy ranged from 5 up to $9 \mathrm{MeV}$ varies from $2 \mathrm{mb}$ up to $3.5 \mathrm{mb}$.

For the definition of $Q_{\alpha}$, let's use the experimental measurements of Radon's and Thoron's concentration in the ground air and at different altitudes in the atmosphere. Data straggling is very wide, as the measurements were made in different times, in different places and by means of different equipments. We have to take into consideration that direct experimental results are presented in the units $\mathrm{Bq} / \mathrm{m}^{3}$. In Table 2, all data is recounted to the units of $Q_{\alpha} \mathrm{cm}^{-3} \mathrm{~s}^{-1}$.

We note the following: the abundance of Radon is determined by the abundance of Radium and its half-life. For Thoron, these values are determined by analogous parameters of Thorium.

Then,

$\frac{\kappa_{\mathrm{Tn}}}{\kappa_{\mathrm{Rn}}}=\frac{M_{\mathrm{Th}}}{M_{\mathrm{Ra}}} \cdot \frac{T_{\frac{1}{2}}(\mathrm{Ra})}{T_{\frac{1}{2}}(\mathrm{Th})}=\frac{8 \cdot 10^{4}}{10^{-10}} \times \frac{1.64 \cdot 10^{3}}{1.39 \cdot 10^{10}}=0.96$

In Eq. (8) $\kappa_{\mathrm{Tn}}$ and $\kappa_{\mathrm{Rn}}$ - are, consequently, the efficiencies of production for Thoron and Radon; $M_{\mathrm{Th}}$ and $M_{\mathrm{Ra}}-$ are the abundances of chemical elements Thorium and Radium, respectively, in the Earth's crust; $T_{1 / 2}(i)$ - are their half-lives periods.

So, as Eq. (8) shows, in average in the Earth's crust efficiency of production of radioactive gases Thoron and Radon are equal.

On the other hand, as it is presented by Serdukova and Kapitanov (1975), the exhalation $q$, that is the output of Thoron and Radon to the ground surface, relate as

$\frac{q_{\mathrm{Tn}}}{q_{\mathrm{Rn}}}=77.8 \frac{C_{\mathrm{Tn}}}{C_{\mathrm{Rn}}}$,

where $C_{i}$ - is the conforming concentrations deep, where gas-exchange between soil and atmosphere does not affect.

The concentration $C_{i}$ of radioactive gas is directly connected with the efficiency of its production $\kappa_{i}$. So, it is clear that near the Earth's crust the main source of $\alpha$-particles of high energy is Thoron. 
In connection with it, it's necessary to note that in the exhaust of radioactive gas the long component (a source of $\alpha$-particles) is Radon, and the short component or shortlived (but, probably, more intensive) is gas Thoron, because $T_{1 / 2}(\mathrm{Rn})=3.825$ days, while $T_{1 / 2}(\mathrm{Tn})=54.5 \mathrm{~s}$.

Of course, all these conclusions break if observations are made near the mines of Uranium ore or in regions of the raised Radium abundance.

There is one more radioactive gas isotope in the Earth's crust - Actinon. It's half-life is $T_{1 / 2}(\mathrm{An})=3.92 \mathrm{~s}$. Actinon is difficult to be observed and therefore it's role can't be distinctly determined.

As it is seen from Eq. (6), if we want to obtain numeric estimation of the role of radioactive gases in the process of production of neutron field near the Earth's surface, it is necessary to know the rate of $\alpha$-particles' production, $Q_{\alpha}$. On the basis of the data of Table 2, we can derive the following conclusion: in the near-Earth layer, the value of $Q_{\alpha} \leq$ $6.4 \times 10^{-5} \mathrm{~cm}^{-3} \mathrm{~s}^{-1}$, excluding Pamir's data (measurements there were made in three different locations), where $Q_{\alpha}$ was increased by a factor of $10^{2}$. Probably, it is connected with the specific geological structure of the region and with increased seismic activity of this zone. Besides, it is obvious, that regions of Uranium mines extremely differ from the mean value according to the value of $Q_{\alpha}$.

In the ground air, the value of $Q_{\alpha}$ can be several ranks higher, than in the near-Earth layer of air. From the data, presented in Table 2, we see that the value of $Q_{\alpha}$ in the soils could be by a factor of $10^{4}$ higher than the analogous value for air in the near-Earth layer.

We note that in the analysis of neutrons' production in soil, it is necessary to take into account the interaction of high-energy $\alpha$-particles not only with nuclei of nitrogen and oxygen, but also with nuclei of other elements of the Earth's crust, mainly, silicon. We take for our calculations of neutrons' production in air, $R_{\alpha}=4.5 \mathrm{~cm}$ and $\sigma_{\alpha j}^{n}=3 \mathrm{mbarn}$, and from Eq. (6) we get

$Q_{n}=7.26 \cdot 10^{-7} Q_{\alpha}$

If now we take $Q_{\alpha}=\sum_{\min }$ and $Q_{\alpha}=\sum_{\max }$, from Table 2, we get:

$Q_{n}=\left(1.31 \cdot 10^{-12} \div 4.64 \cdot 10^{-11}\right) \mathrm{cm}^{-3} \mathrm{~s}^{-1}$

For the Pamir region, we obtain $Q_{n} \geq 10^{-9} \mathrm{~cm}^{-3} \mathrm{~s}^{-1}$, while for the region of Uranium mines $Q_{n}=4 \times$ $10^{-7} \mathrm{~cm}^{-3} \mathrm{~s}^{-1}$.

If we want to find out the role of this source, we note that the mean energy of neutrons, produced by the studied mechanism, is $E \approx 1 \mathrm{MeV}$. The lifetime of neutrons of such energy in the lower atmosphere, $\tau_{n}$ consists of thermalisation time, $t$ and capture time of thermal neutron, $t_{a}=1 / n v \sigma=0.07 \mathrm{~s}$, where $n-$ is the concentration of nuclei of nitrogen, $v=$ $2200 \mathrm{~m} / \mathrm{s}$ - the velocity of thermal neutron, $\sigma=1.8$ barn the cross-section of capture of thermal neutron by nitrogen.

The thermalisation time can be estimated according to the theory of neutrons' transmission in the medium (Bekurtz and
Virtz, 1968) by the equation

$t=\frac{1}{\xi n \sigma v_{a v}} \ln \frac{E_{0}}{E_{t} h}$,

where $E_{0}$ - is theinitial energy of a neutron; $n$ - is the concentration of atoms in air; $\sigma=10$ barn (Adamchuk, 1995) - the cross section of neutron's scattering in air; $\xi=0.14$ (Volodichev et al., 1990) for air; $v_{a v}$ - is the mean velocity of a neutron. Here, $v_{a v}$ is the most vague value in the equation for $t$. We take $E_{0}=0.45 \mathrm{eV}$ to diminish vagueness, that is, $E_{0}$ is equal to the energy of cadmium cutting for our particular experiment. Then, $t=0.11 \mathrm{~s}$ and $\tau_{n}=t_{a}+t \approx 0.2 \mathrm{~s}$.

Taking $Q_{n}$ and $\tau_{n}$ into consideration, we obtain according to Eq. (7):

$N_{n}=\left\{\begin{array}{l}\left(2.6 \cdot 10^{-13} \div 9.3 \cdot 10^{-12}\right) \mathrm{cm}^{-3} \\ 2 \cdot 10^{-10} \mathrm{~cm}^{-3}-\text { Pamir } \\ 8 \cdot 10^{-8} \mathrm{~cm}^{-3}-\text { Uranium mines }\end{array}\right.$

Comparing these values with the concentration of neutrons with energy $E \leq 0.45 \mathrm{eV}$, which was found experimentally $N_{n}^{\text {exp }}=4.8 \cdot 10^{-10} \mathrm{~cm}^{-3}$, we obtain:

$\frac{N_{n}}{N_{n}^{\exp }}=\left\{\begin{array}{l}\leq 2 \% \\ \approx 50 \%-\text { Pamir } \\ >100 \%-\text { Uranium mines }\end{array}\right.$

Of course, in ideal case, if somebody wants to estimate the contribution of radioactive gases from the Earth's crust into neutrons' concentration observed near the Earth's surface, he have to compare the values from Eq. (11) with the concentration (or with flux) of neutrons, which are produced by the interaction of cosmic rays with the lower atmosphere. But it is clear that in that case the value of the contribution of radioactive gases will only increase.

We can conclude from Eq. (12) that contribution of radioactive gases into experimentally observed concentration of neutrons with $E \leq 0.45 \mathrm{eV}$ covers a wide range from several up to one hundred percents.

On the other hand in the ground air as it is seen from Table 2, $Q_{\alpha}$ is several times higher than in the near-Earth layer of air. As a rule, information about radioactive gases in the ground air is obtained for the depth of about $1 \mathrm{~m}$. It is obvious, that $\alpha$-particles with high energy, which are produced by the decay of radionuclides at this depth interact with nuclei of chemical elements composed the ground and also produce neutrons. In average, half of produced neutrons will go out into the atmosphere. Just existence of neutrons' flux directed from the depth of the ground to the atmosphere can explain anisotropy of neutrons' flux near the Earth's surface, which has been discussed above.

We can use Eqs. (6) and (7) and the relation between the concentration and the flux to calculate the flux of neutrons.

There are a lot of different types of ground. We review the most popular - sandy and clayey. Their density $\rho=$ $1.5 \mathrm{~g} / \mathrm{cm}^{3}$. 
Table 3. Calculated neutron flux $\left(F_{n}^{\mathrm{cal}}\right)$ on different types of ground

\begin{tabular}{lcc}
\hline Ground type & $F_{n}^{\min \mathrm{cm}^{-2} \mathrm{~s}^{-1}}$ & $F_{n}^{\mathrm{max}} \mathrm{cm}^{-2} \mathrm{~s}^{-1}$ \\
\hline Caolinite & $10^{-7}$ & $1.4 \times 10^{-4}$ \\
Montmorillonite & $1.4 \times 10^{-7}$ & $2.6 \times 10^{-4}$ \\
Sand & $5.0 \times 10^{-7}$ & $9.0 \times 10^{-4}$ \\
\hline
\end{tabular}

From the point of view of chemical composition, sand consists of silicon oxide $\mathrm{SiO}_{2}$, and clay has more complicated composition. One type of clay is caolinite $\mathrm{Al}_{4}\left(\mathrm{Si}_{4} \mathrm{O}_{10}\right)(\mathrm{OH})_{8}$, and the second is montmorillonite $\mathrm{Si}_{8} \mathrm{Al}_{4} \mathrm{O}_{20}(\mathrm{OH})_{4}$.

So, while calculating the neutrons' flux from the Earth's crust, we have to take into account the production of neutrons during the interaction of $\alpha$-particles with the nuclei of silicon, aluminum and oxygen. The life-time of a neutron in the ground is determined by its capture with nuclei of silicon, aluminum and hydrogen. Results of calculated neutron flux on different types of ground are presented in Table 3.

Minimum and maximum fluxes of neutrons are determined by the minimum and maximum value of $Q_{\alpha}$. Value of $R_{\alpha}$ of $\alpha$-particle's mean-free path in the ground was selected as mean-free path in silicon (Mineev, 1990) for $\alpha$-particle with $E_{\alpha}=5 \mathrm{MeV}$ taking into consideration the ground's density, $R_{\alpha}=40 \mu \mathrm{m}$.

We note that the ratio $F_{n}^{\mathrm{cal}} / F_{n}^{\exp }$ for different types of ground is in agreement with the contribution of the Earth's crust as a source of neutrons into the total flux of thermal and slow neutrons during the quiet helio- and geophysical periods, taken from the information about anisotropy.

\section{Conclusions}

1. Data of baloon experiments lead to a conclusion, that up to altitute of $1.5-2 \mathrm{~km}$ there is no vertical control traverse of neutron counting rate.

2. From the DAIZA experimental data we can conclude that the neutron flux near the Earth's surface depends on the season and for quiet periods of measurements it varies from $-20 \%$ up to $20 \%$ depending on the month.

3. During the splashes of neutron counting rate the value of anisotropy of neutron flux can be close to $100 \%$.

4. Obtained calculation and experimental results show that radioactive gases in the upper layers of the Earth's crust and in the air, provide essential (in some places up to $100 \%$ ) part of neutron flux in the range of thermal and slow neutrons near the Earth's surface.
5. The mean life-time of a neutron with energy having near the Earth's surface is $\tau_{n}=0.2 \mathrm{~s}$, then, at velocity $v_{a v}=6 \times 10^{5} \mathrm{~cm} / \mathrm{s}$, the mean distance, which neutron will cover is $l=v_{c p} \times \tau_{n}=1200 \mathrm{~m}$. The capture path of thermal neutrons in the air is only several hundred meters.

All obtained results lead to a conclusion that the Earth's crust is an essential source of neutrons of which the total neutron flux varies both in space and in time, depending both on conditions of the atmosphere and the Earth's crust itself and different geodynamical phenomena in the Earth's crust.

The most important dynamical phenomena in the Earth's crust are earthquakes, mud flows and volcano activity. Obtained results allow to use observations of thermal neutron flux variations for methods of short-term prediction of natural hazards.

\section{References}

Adamchuk, Yu. V.: Atlas of effective neutron cross sections, M., 1995.

Anderson, W., Moynerod, W. V. and Turneng, R. C.: Nature, 174, 424, 1954

Bekurtz, K. and Virtz, K.: Neutron physics, M., Atomizdat, 1968.

Beliaeva, E. A., Kuzhevskij, B. M., and Nechaev, O. Yu.: Physical basis of a forecasting method for earthquakes and volcano activity according observation of variations of neutron flux near the Earth crust, General Assemble IUGG-99, Birmingham, 1999a.

Beliaeva, E. A., Kuzhevskij, B. M., Nechaev, O. Yu., and Panasyuk, M. I. Neutron sources and nature of time variations of neutron flux near the Earth surface, Physical Ecology, Moscow, 4, 89, 1999b.

Burian, I.: Ann. de l'Assosiation belge de Radioprotection, 21, 1, 39, 1966.

Gorshkov, G. V., Zabkin, V. A., Lyatkovskaya, N. M., and Tsvetkov, O. S.: Natural neutron background of the atmosphere and the Earth's crust, M., Atomizdat, 1966.

Kapitonov, I. M. and Gabelko, A. S.: Report of INP MSU, 1990.

Kuzhevskij, B. M.: Nuclear processes in the atmosphere of the Sun and solar cosmic rays, Energoatomizdat, 72, 1985.

Kuzhevskij, B. M.: Nucleonics-based monitoring of the ecological environment balance of the Earth crust and lower atmosphere, Internet-site: http://srd.sinp.msu.ru/bmk/nuclear.doc, 2000.

Kuzhevskij, B. M., Nechaev, O. Yu., and Shavrin, P. I.: Thermal neutron anisotropy in the Earth atmosphere, Geomagnetism and Aeronomy, 35, 2, 166, 1995.

Kuzhevskij, B. M., Nechaev, O. Yu., Shavrin, P. I., Beliaeva, E. A., and Sirotkin, I. I.: Variations of neutron concentration in the field of thermal neutrons of the Earth crust, INP MSU 96-7/414, 1996.

Lingenfelter, R. E., Flamm, E. T., Canfield, E. H., et al.: Highenergy solar neutrons. I. Production in flares, J. Geophys. Res., 70, 4077-4086, 1965.

Mineev, Yu. V.: Space Research. Scientific and technological results. VINITL, 32, 118, 1990.

Rudakov, V. P., Voitov, G. I., Kurkov, O. A., and Chaika, V. P.: Reports of Russian Academy of Sciences, 343, 389, 1995.

Serdukova, A. S. and Kapitanov, Yu. T.: "Rn isotopes and their decomposition products in nature”, M., Atomizdat, 1975. 
Volodichev, N. N., Kuzhevskij, B. M., Nechaev, O. Yu., and Panasyuk, M. I.: Registration of neutrons and gamma-quants in baloon experiments, Preprint INP MSU 90-3/149, 1990.

Volodichev, N. N., Kuzhevskij, B. M., Nechaev, O. Yu., and Panasyuk, M. I.: Strong increasing of neutron flux during the solar eclipse 22.07.1990, Space Research, 30, 422, 1992.

Volodichev, N. N., Kuzhevskij, B. M., Nechaev, O. Yu., Panasyuk, M. I., and Podorolskij, A. N.: Astrogeophysical reasons for splashes of neutron irradiation intensity and seismoactiveness on the Earth, Preprint INP MSU 2001-5/645, 2001. 